\title{
CONTEXTO POLÍTICO DEL FUTURO DE LOS BOSQUES: PANDEMIA, ECONOMÍA Y AMBIENTE EN EL PERÚ
}

\author{
Patricia Iturregui Byrne \\ Investigadora independiente
}

\begin{abstract}
Resumen: ¿Cómo se relaciona la política nacional de competitividad con la sostenibilidad ambiental en el Perú? ¿Se ha cumplido con las recomendaciones de la OCDE para el desempeño ambiental del Perú en el tema de economía y ambiente? ¿Cómo puede revertirse la deforestación de la Amazonia según la opinión de expertos y los ex ministros del Ambiente? Estas son las preguntas que trata de responder este artículo, señalando también áreas de posible investigación académica futura.
\end{abstract}

Palabras clave: pandemia, competitividad, deforestación, OCDE y Perú, planes nacionales, cambio climático, diversidad biológica, inversión privada forestal, plantaciones. 


\title{
Policy context for the forest in the future: Pandemic, economy and environment in Peru
}

\begin{abstract}
How is the national competitiveness plan connected to sustainability in Peru? The OECD recommendations for environmental performance in Peru related to economy and environment has been pursued? How to reverse the Amazon deforestation in Peru according to experts and ex ministers of the Environment? These are the questions that this article aims to answer pointing out which are the areas for future academic research.
\end{abstract}

Keywords: pandemic, competitiveness, deforestation, OCDE and Peru, national plans, climate change, biological diversity, private investment in forests, plantations.

\section{Patricia Iturregui Byrne}

Abogada por la PUCP, master en Derecho Ambiental por la Universidad de Londres y doctoranda por la Universidad de Colonia/Alemania. Miembro del roster de expertos de la Convención de Naciones Unidas sobre Cambio Climático en el tema de políticas y medidas. Miembro del Grupo de Investigación de Derecho Ambiental (GIDAMB- INTE PUCP). Consultora ambiental independiente y catedrática de derecho ambiental.

Correo: patricia.iturregui@pucp.edu.pe 


\section{Introducción}

Según el reporte del Programa de Ambiente de las Naciones Unidas sobre enfermedades zoonóticas de julio de 2020 (UNEP-ILRI 2020), cerca del $75 \%$ de las enfermedades infecciosas del ser humano provienen del «salto» de trasmisores de especies animales hacia los humanos.

El referido reporte menciona diversas causas antropogénicas a la emergencia de zoonosis, destacando la deforestación - a la que se atribuye la emergencia del ébola en África Occidental一, la intensificación no sostenible de la agricultura, la demanda de proteína animal, así como el cambio climático.

El consenso científico es que debe invertirse recursos en enfrentar estos problemas estructurales que están ocasionando la recurrencia de enfermedades zoonóticas y pandemias.

Una afirmación del reporte, ya conocida entre los expertos en zoonosis, es que los factores causantes de la pandemia son los mismos que los causantes del cambio climático y la pérdida de biodiversidad, desafíos de largo plazo que no se han superado con la pandemia del COVID-19.

Sin referirnos al detalle sobre cada uno de estos factores causantes de la zoonosis, baste mencionar cómo ha crecido la demanda mundial de proteína animal en los últimos cincuenta años, siendo que la producción de carne aumentó en $260 \%$. Esta tendencia ha generado que, en la actualidad, del 100\% de mamíferos en el mundo, el $60 \%$ es ganado, el 36\% humano y apenas el 4\% es de mamíferos silvestres (BarOn et al. 2018), lo que tiene importantes consecuencias en la estabilidad de los ecosistemas y el clima mundial. Esto ha permitido aseverar que el Bos Taurus y el Homo Sapiens son ahora los vertebrados dominantes en la biósfera (Smil 2016).

Respecto de la intensificación no sostenible de la agricultura, esta se asocia a más del $50 \%$ de enfermedades zoonóticas que han emergido en humanos. Es de notar que la tercera parte de las tierras de cultivo se usan para alimentar animales y esto puede conducir a la deforestación.

De otro lado, el cambio climático cada vez más influye en la distribución geográfica y abundancia de especies, tales como murciélagos, monos y roedores, incluyendo aquellos con patógenos, así como mosquitos y otros vectores que trasmiten diversas enfermedades como el dengue y la chikunguya. 
En Brasil se ha revelado el vínculo entre brotes de enfermedades infecciosas y eventos climáticos extremos, como El Niño y La Niña, olas de calor, sequías e inundaciones, cuya mayor frecuencia es atribuible al cambio climático, la fragmentación del hábitat y la deforestación.

Migrar de las respuestas de corto plazo a compromisos de largo plazo que aseguren la salud humana, del planeta y la vida silvestre es indispensable. Reconocer que la salud es una, esto es, que la salud humana, la salud de la vida silvestre y del planeta están irremisiblemente vinculadas, es el consenso científico actual. Este enfoque, denominado también One Health (Simpleshow Foundation 2017), sugiere agendas comunes de investigación y acción entre las universidades, el Estado e inversionistas privados.

Es urgente actuar decididamente para enfrentar los factores causantes de la pandemia actual y de otras pandemias que puedan sobrevenir. Queda claro que en los próximos doce meses los esfuerzos estarán dedicados a la recuperación económica y las medidas para dominar la pandemia, pero no hay que perder de vista los problemas estructurales que debemos enfrentar como la deforestación, el uso no sostenible de la agricultura y la informalidad laboral, entre otros. Hay estudios dispersos que sugieren que estas situaciones pueden transformarse en oportunidades para el Perú.

Este artículo está dividido en siete partes. En la primera se presentan unas notas sobre cómo a través del tiempo se ha dado el vínculo entre política ambiental y competitividad en nuestro país. Mientras que en la segunda se toca el tema del desempeño ambiental y el ingreso de Perú a la OCDE; y en la tercera se hace referencia a la competitividad e informalidad en relación al tema de bosques; mientras que en la cuarta parte se habla de la situación actual de los bosques en el país. En la quinta parte se considera la problemática de la deforestación y la necesidad de crear empleo, así como los ejes que permitirían un impulso del desarrollo sostenible de la Amazonia; en la sexta parte se toca brevemente la deforestación cero como alternativa a futuro. Se cierra con unas reflexiones finales. Asimismo, a la investigación bibliográfica se le suma la realización de una serie de entrevistas, llevadas a cabo en la primera semana de setiembre de 2020, para enriquecer el artículo, para ello se entrevistó a cuatro ex ministros del Ambiente y a un asesor financiero de inversiones extranjeras forestales. 


\section{Un recuento sobre política ambiental y competitividad en el Perú}

Desde 1979 el Foro Económico Mundial mide la competitividad de los países. La definición que usa es la de «un conjunto de instituciones, política y factores que determinan el nivel de productividad de un país» ${ }^{1}$.

El profesor y economista liberal Michael Porter ha venido afirmando desde 1995 (Porter y van der Linde 1995) la relación positiva entre regulación ambiental e innovación. Las empresas realmente pueden beneficiarse con regulaciones ambientales adecuadamente diseñadas que sean más estrictas que en otros países o aprobadas en una etapa temprana. Estimulando la innovación, las normas ambientales pueden aumentar la competitividad. En otros contextos, señala Porter, los economistas tienen mucho interés en argumentar que el cambio tecnológico ha contradicho predicciones de amplios y severos costos ambientales.

El comentario inmediato puede ser que no somos un país desarrollado y no podemos tener los estándares ambientales de estos países, a lo que puede responderse que tampoco se trata de ser el «botadero» de tecnologías obsoletas, tales como plantas de energía usadas o vehículos de segunda mano².

Porter indica que la contaminación está muy vinculada al uso ineficiente de los recursos naturales y factores de producción, o con pérdidas energéticas. En suma, reducir la contaminación mejora la productividad.

Desde un enfoque jurídico, hay una conexión entre política ambiental y competitividad en nuestra legislación ambiental. Si bien merece un artículo a parte, dejo al lector la información de la Ley General del Ambiente (2005), que manda que toda política pública debe articularse a los objetivos de protección ambiental y obliga al Estado y a las universidades a promover «La diversificación y competitividad de la actividad pesquera, agraria, forestal y otras actividades económicas prioritarias ${ }^{3}$. Siendo el texto en sí una afirmación general, la exposición de motivos de la referida ley no contiene detalles, ejemplos, ni explicaciones del vínculo entre políticas y medidas ambientales con el incentivo a la competitividad.

En 2008, a iniciativa del Poder Ejecutivo, facultado para legislar respecto de la implementación del Acuerdo de Promoción Comercial Perú - Estados Unidos, se crea el Ministerio del Ambiente. La parte considerativa del decreto legislativo 1013 señala la necesidad de crear el ministerio para «materializar el apoyo a la competitividad económica para el aprovechamiento del Acuerdo Comercial». Por ello, uno de los objetivos específicos del MINAM es el contribuir a la competitividad del país a través de un desempeño ambiental eficiente ${ }^{4}$.

\footnotetext{
A nivel empresa, según diversos autores, los indicadores clave son (i) productividad, (ii) ganancia, (iii) presencia en el mercado e (iv) inversión en innovación, investigación y desarrollo, ver Dresch et al. (2018) y Cann (2017).

2 Ver el tema de trasferencia de la contaminación en vez de trasferencia de tecnologías en Iturregui y Dutschke (2005: 13).

3 Artículo 124.1 de la Ley General del Ambiente.

4 Artículo 3.2.d del decreto legislativo 1013.
} 
El referido acuerdo comercial incluye un capítulo ambiental que compromete al Perú a que sus leyes proporcionen y estimulen altos niveles de protección ambiental. El referido instrumento jurídico internacional ratifica implícitamente los derechos existentes en la Ley General del Ambiente referidos al derecho a la información ambiental, así como el derecho a la justicia ambiental, esto es que las personas puedan solicitar a las autoridades que investiguen infracciones ambientales y a que los procedimientos judiciales o administrativos estén disponibles para sancionar o reparar las infracciones ambientales. Para esto se crea un Consejo de Asuntos Ambientales que vigila el cumplimiento del capítulo ambiental con participación del público interesado. El Acuerdo incluyó un anexo forestal para combatir la tala ilegal, así como una modificación al Código Penal para incrementar las penas al tráfico de vida silvestre y comercio ilegal de madera.

Habiéndose avanzado en el marco jurídico forestal por impulso del Acuerdo Comercial, no puede decirse que la tala ilegal, así como el comercio ilícito de fauna silvestre, haya disminuido de forma significativa ${ }^{5}$.

Siguiendo en el tiempo, en $\mathrm{CADE}^{6}$-Perú 2010 el profesor Michael Porter manifestó que, si bien las políticas macroeconómicas han sido sólidas por la apertura del comercio, no todos se han beneficiado del crecimiento económico ya que impulsa sectores que no generan empleo. Porter recomendó enfáticamente que Perú genere más valor con los recursos naturales por su potencial de convertirse en un hub regional. Asimismo, aseveró que la «prosperidad continúa siendo baja y grandes sectores de la población no tienen acceso a servicios básicos» ${ }^{7}$.

La pandemia ha puesto en evidencia la certeza de los comentarios de Porter: las inversiones en el sistema de salud peruano son una de las más bajas de la región. Siendo conocida la simpatía que genera las propuestas de Porter en el sector visionario del empresariado peruano, llama la atención que estos no hayan influido en la dirección correcta en los gobiernos ${ }^{8}$.

Porter añadió, en el CADE 2010, que Perú necesita tener claro que el siguiente paso es el incremento de la competitividad y contar con una visión estratégica en materia económica con objetivos para los próximos diez años, que tenga entre sus componentes focos de desarrollo regionales lejos de Lima.

El impulso a la inversión privada en procesos productivos que utilicen tecnologías e insumos que reduzcan las emisiones de carbono e impactos ambientales, así como la reconversión de las industrias contaminantes, es lo que teóricamente considera el MEF como ecoeficiencia en la primera Agenda Nacional de Competitividad (de corto plazo) para el 2012-2013.

La claridad de este enfoque, sin embargo, no es muy consistente con las metas ambientales que la referida agenda contiene, las que son relativamente modestas y poco transformadoras. Aún más, el objetivo de fortalecer capacidades

\footnotetext{
5 Ver Reporte de Verificación del Timber Committee (2018).

Conferencia Anual de Ejecutivos.

Para mayor información ver las declaraciones de Michael Porter (Conexión ESAN 2010).

8 Michael Porter ha sido invitado varias veces al Perú para los CADE. En octubre de 2020 realizó una presentación central en el evento Perú Sostenible (Comunicaciones Perú2021 2020).
} 
empresariales para incrementar niveles de productividad no involucra la gestión ambiental.

Llama la atención que la referida agenda no contenía metas para promover la industria forestal, habida cuenta que la Ley forestal (2011) incluía el mandato de promover el acceso de los productos forestales a servicios financieros, a mercados nacionales e internacionales, y mejorar las condiciones de competitividad del sector. Cabe mencionar que la reglamentación de la ley demoró cuatro años.

A este punto, puede decirse que si bien la gran mayoría tiene claro que el desarrollo sostenible se cimienta en tres pilares - como son el desarrollo económico, social y ambiental-, en el Perú no se ha internalizado el principio generalmente aceptado de que la política ambiental puede acrecentar la competitividad. Esa falta de visión obstaculiza la acción concertada del MEF y el Ministerio del Ambiente.

Sin embargo, como dijo Porter recientemente en el evento Perú Sostenible 9 , bien puede el sector privado liderar el cambio por ser la única institución que produce riqueza y prosperidad. El valor compartido es el concepto clave en base a tres constataciones básicas:

- $\quad$ las necesidades sociales representan las mayores oportunidades de mercado que no están cubiertas;

- $\quad$ los problemas ambientales y sociales crean costos económicos a las empresas;

- las debilidades de la comunidad donde operan los negocios afectan la productividad y las ganancias.

Con riesgo de redundancia, no hay que olvidar que hoy se habla de competitividad sostenible (Andreoni y Miola 2016: 37), definida como el «conjunto de instituciones, políticas y factores que hacen a una nación productiva en el largo plazo asegurando la sostenibilidad social y ambiental». Lo importante de esto es la conexión directa con muchas de las metas de la agenda de los objetivos de desarrollo sostenible de las Naciones Unidas.

\section{El desempeño ambiental y la voluntad del Perú de ingresar a la OCDE}

En 2014, el Perú suscribe el Programa País con la $\mathrm{OCDE}^{10}$, con cinco ejes principales, siendo uno de ellos el fortalecimiento de los estándares ambientales ${ }^{11}$, con la intención de iniciar el proceso de ser miembro en el mediano plazo.

\footnotetext{
9 Ver presentación de Michael Porter en Comunicaciones Perú 2021 (2020).

10 Organización para la Cooperación con el Desarrollo Económico. Los países de América Latina miembros de la OCDE son Chile, Colombia, Costa Rica y México.

11 Los otros ejes son: i) identificación de barreras al crecimiento y desarrollo, ii) mejora de la institucionalidad y gobernanza pública, iii) transparencia del Estado y la lucha contra la corrupción y iv) fortalecimiento de la productividad y el capital privado.
} 
Los temas ambientales más destacables del Programa País fueron dos: la adhesión a la Declaración de Crecimiento Verde (2009) de la OCDE —realizada en 2015- y la realización de una evaluación de expertos internacionales del desempeño ambiental del Perú de los últimos diez años.

Lo esencial de la Declaración de Crecimiento Verde de la OCDE es la propuesta que lo «verde» y el «crecimiento» interactúen mutuamente. Esto es, el impulso al crecimiento económico y el desarrollo deben garantizar que los recursos naturales activos continúen proporcionando el abastecimiento y los servicios ambientales de los que depende nuestro bienestar. Se señala como temas prioritarios el agua, la biodiversidad y el cambio climático.

La Declaración de Crecimiento Verde de la OCDE recomienda:

a. promover inversión verde con instrumentos de mercado y otras políticas para cambiar las conductas y producir respuestas apropiadas del sector privado, considerando la expansión de incentivos;

b. reforma de políticas de subsidios a los combustibles fósiles o a la producción que incremente las emisiones de gases;

c. trabajar reglamentaciones y políticas que aseguren señales de precios a largo plazo que estimulen resultados ambientales eficientes.

El avance respecto de estas recomendaciones en el Perú es modesto. Si bien el proyecto PAGE (2017) ${ }^{12}$ otorgó importantes insumos, el diálogo Gobiernosector privado no ha dado resultados o no ha sido fluido. En el marco de la Alianza del Pacífico, se creó una Plataforma de Crecimiento Verde que sentaba a la mesa a los ministros de Ambiente y líderes empresariales, pero el denominado «Grupo de Medio Ambiente y Crecimiento Verde», creado en junio de 2016, no ha funcionado.

Se cuenta con ocho lineamientos de crecimiento verde aprobados mediante resolución ministerial 161-2016 MINAM, donde se menciona que está en elaboración una estrategia nacional de crecimiento verde, pero esta parece no haber sido concluida ni puesta en consulta.

Respecto de la evaluación de desempeño ambiental, el estudio realizado por la OCDE/CEPAL realizó 66 recomendaciones, para lo que se aprobó, con el decreto supremo 005-2017-MINAM, un plan de acción que ha sido objeto de 2 reportes de seguimiento. A continuación, resumimos las 7 recomendaciones para el tema de economía y ambiente que tienen relación con la Declaración de Crecimiento Verde, indicándose su nivel de cumplimiento.

12 PAGE es un proyecto liderado por la Organización Internacional del Trabajo para trabajar con el Gobierno peruano las políticas públicas de crecimiento verde para cumplir con el Acuerdo de Paris sobre cambio climático. 


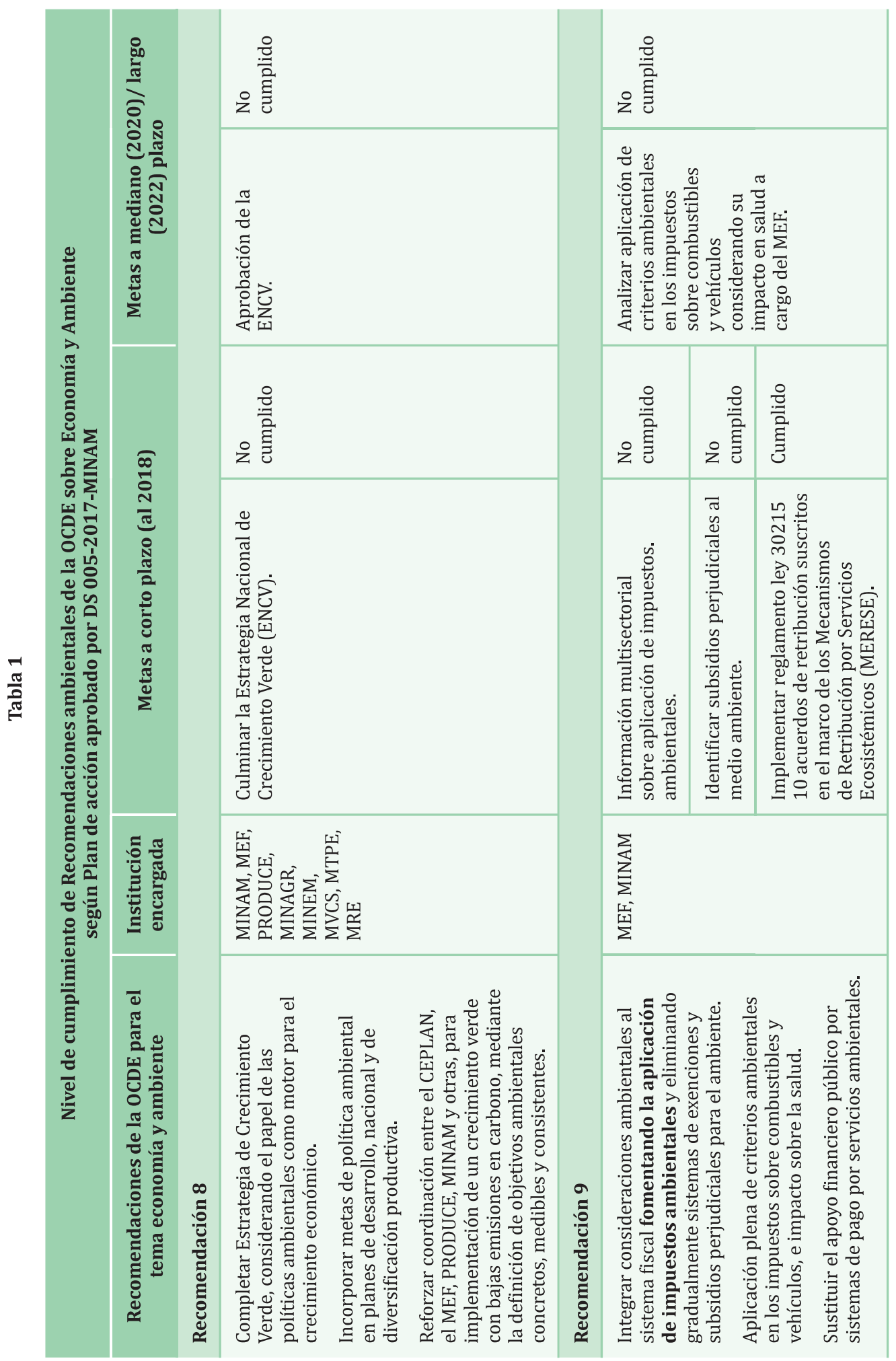




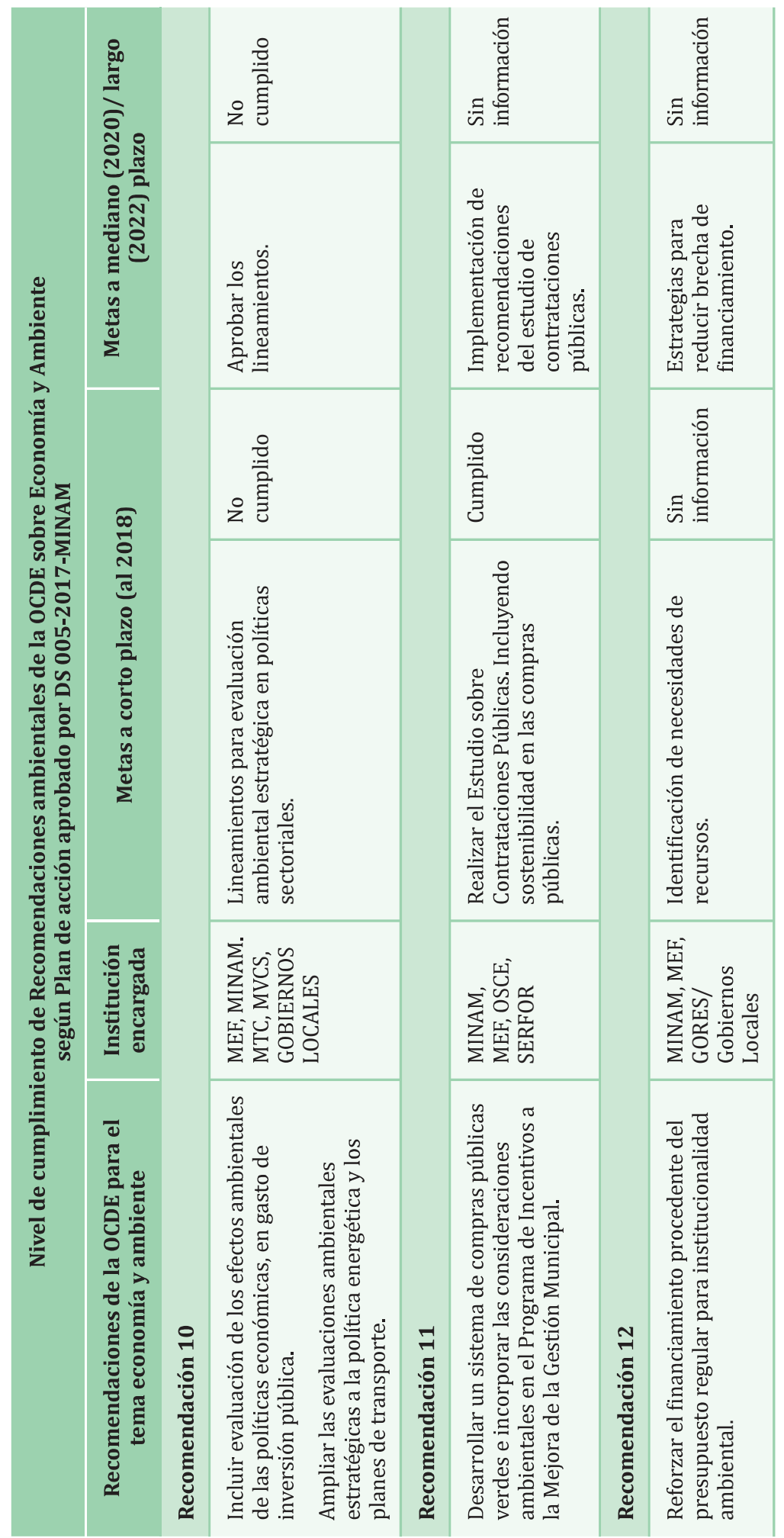




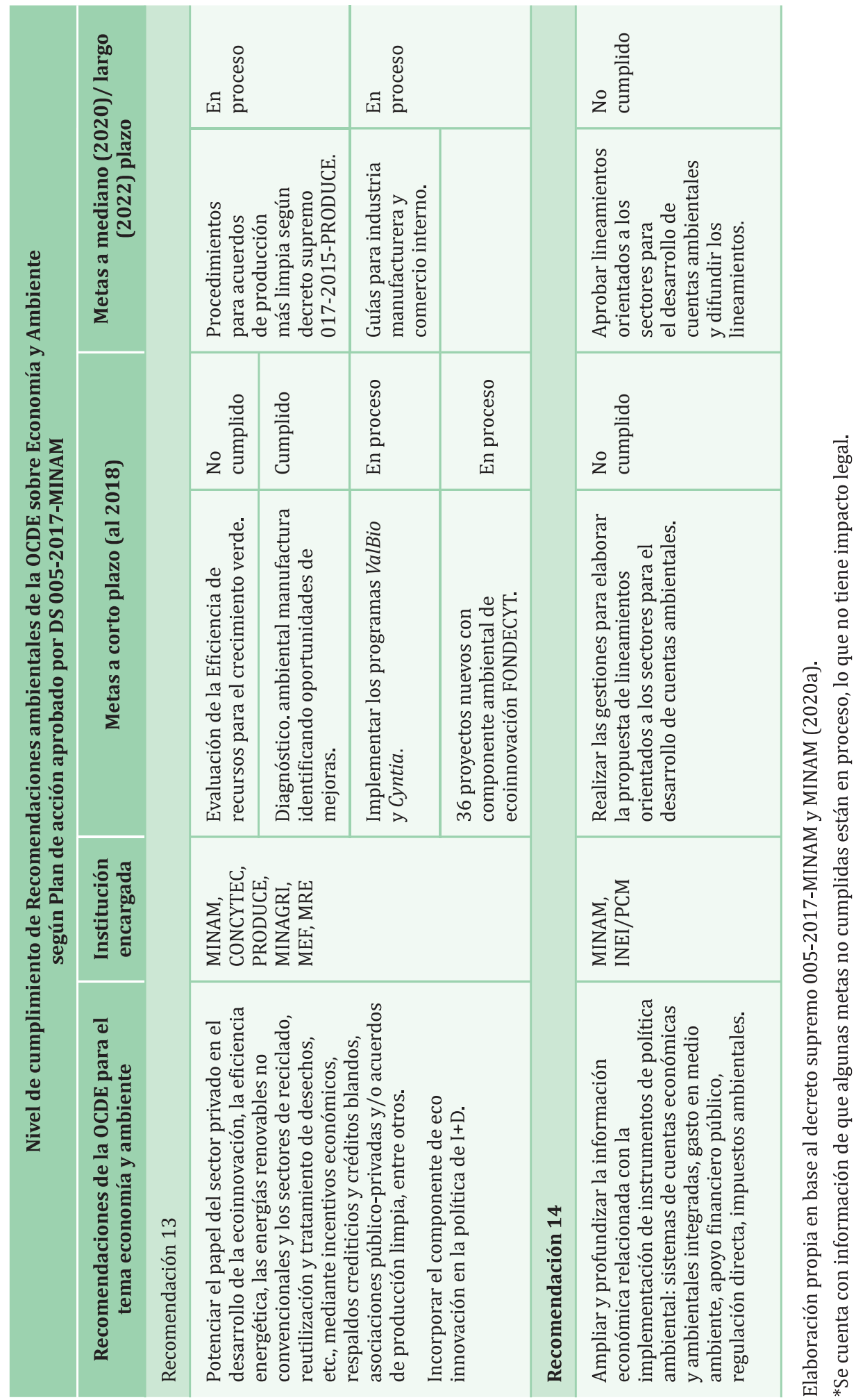


Solo a fines de 2019 el Gobierno aprobó el Plan Nacional de Competitividad 2019-2030 ${ }^{13}$ que constituye una positiva coincidencia de fechas tanto de los Objetivos de Desarrollo Sostenible como del Acuerdo de Paris sobre cambio climático, donde el cumplimiento de las metas de control de emisiones de gases que cada país ha ofrecido culmina en 2030.

De los nueve objetivos, el Plan responsabiliza al MINAM del objetivo de Sostenibilidad Ambiental para «una mejor utilización de nuestros recursos naturales, (...) métodos de producción y patrones de consumo que fomenten (...) una "economía circular", generando así una variedad de efectos positivos sobre la competitividad peruana». Adicionalmente, en el objetivo 4, referido al impulso de mecanismos de financiamiento local y externo, se incluye la medida de instrumentos financieros verdes, que involucra la Hoja de Ruta de Requerimientos para la adecuación técnica y legal orientada a la emisión de bonos verdes (diciembre de 2019) y la Emisión del Primer Bono Soberano Verde del Estado Peruano para julio de 2021.

Siendo que los factores causantes de la pandemia son los mismos que los causantes del cambio climático y la pérdida de biodiversidad, toca ahora describir cada una de las medidas del referido plan. Estas son las siguientes: 


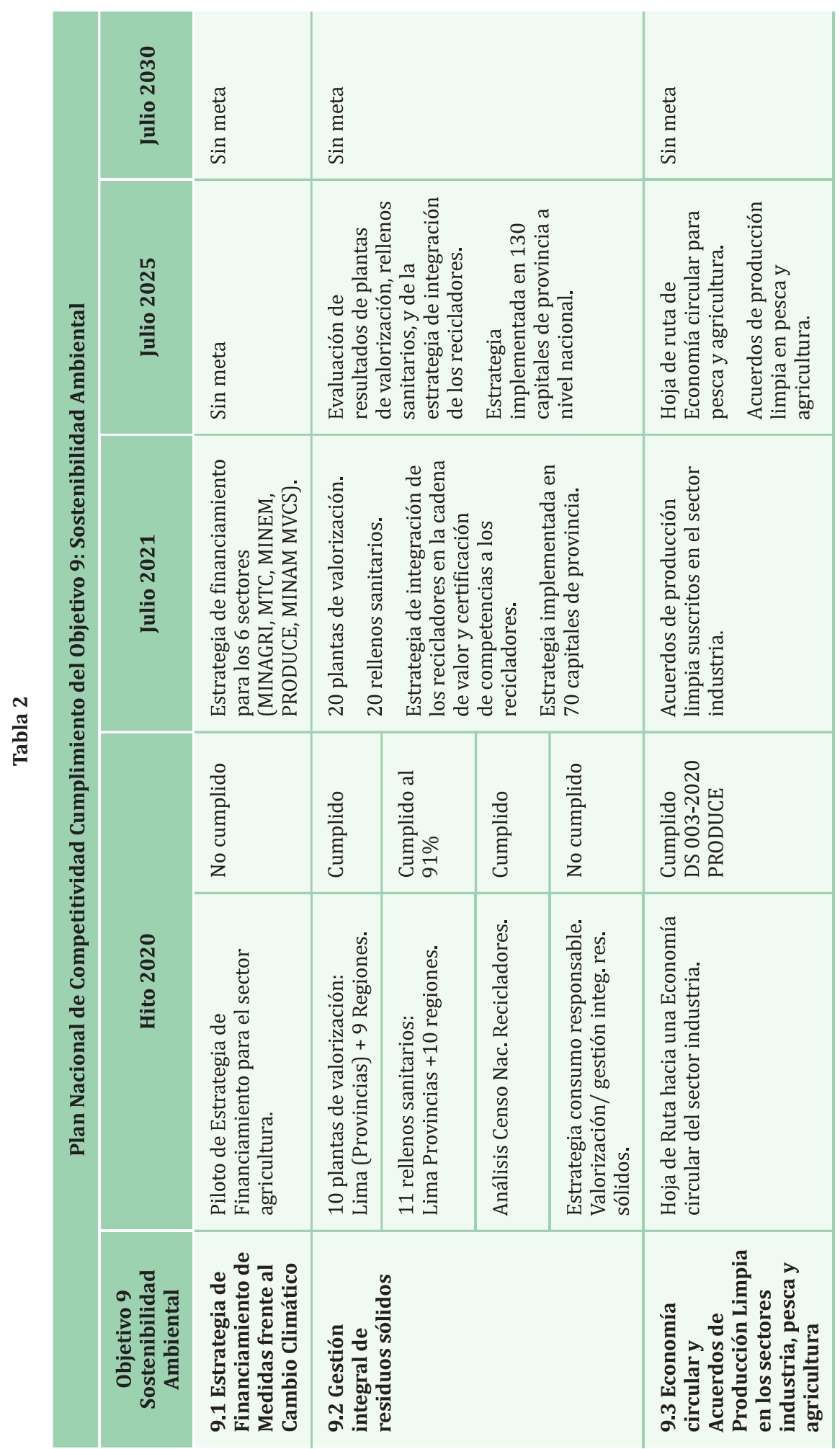




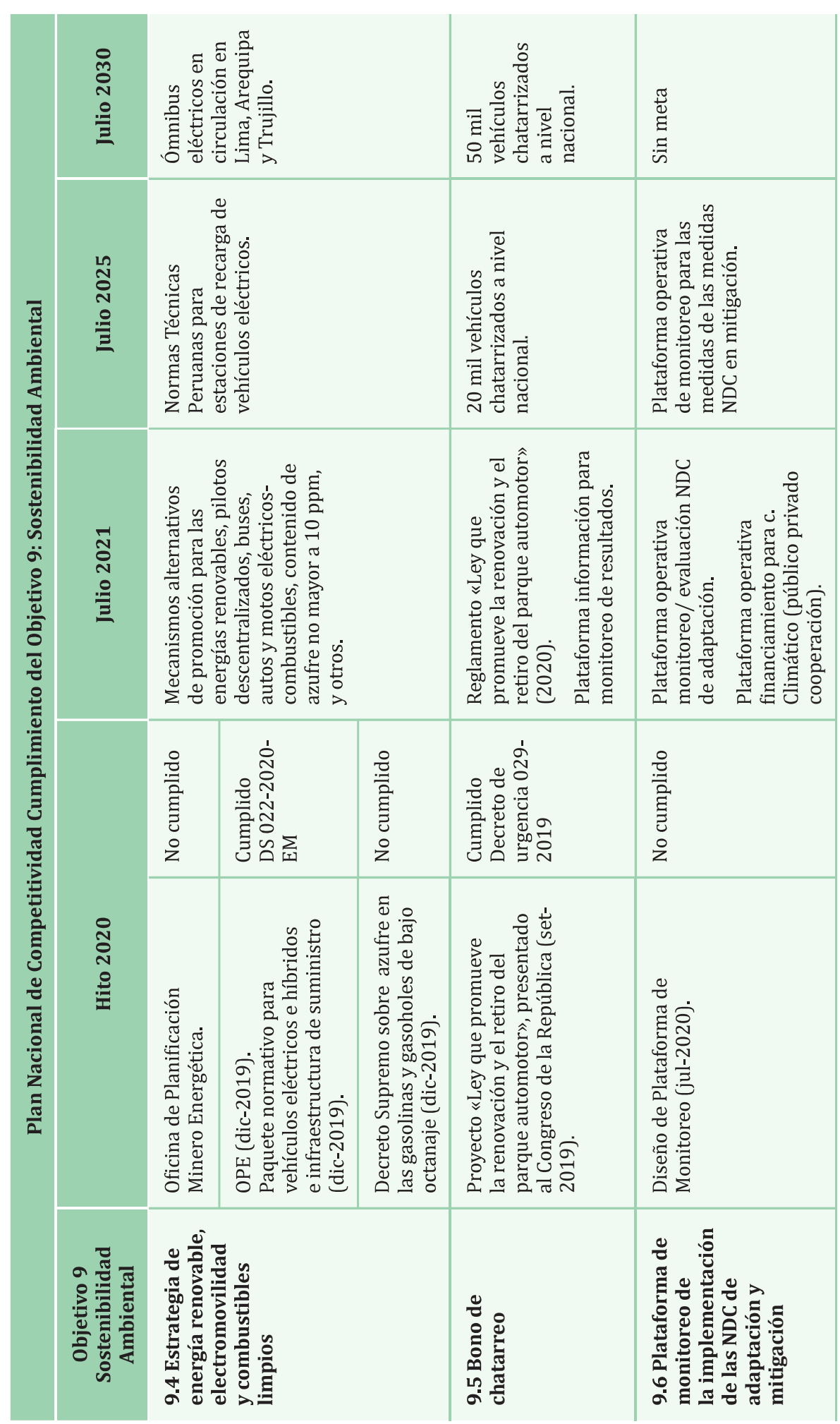


El balance del Plan Nacional de Competitividad es de un cumplimiento parcial de las metas comprometidas para el primer semestre de 2020. Lo que llama la atención es la ausencia de metas de largo plazo en algunos casos. Respecto del cambio climático, bien podría haberse incluido los hitos sugeridos por el propio compromiso asumido para 2030 por el Estado peruano, o los avances de los planes de carbono neutralidad al 2050 alentados por el Acuerdo de París. Otra meta de mediano plazo podría ser la incorporación de los bosques naturales a las cuentas nacionales, implicando que el patrimonio de la nación disminuya con su destrucción, en lugar de aumentar con la deforestación.

Es importante notar que el Fondo Verde para el Clima (GCF) es uno de los fondos internacionales más importantes en el tema de cambio climático, y el MEF ha pasado a ser el punto focal de este fondo desde hace más de siete años. Esto significó suprimir la función que desempeñaba el Profonanpe, con quien se avanzó en la formulación y aprobación del primer proyecto del GCF en un proyecto amazónico. Desde que el MEF asumió el rol de punto focal no hay ningún proyecto nuevo en ejecución y el Perú ha perdido las oportunidades que dicho fondo ofrece al país, incluyendo oportunidades para el sector privado.

\section{Competitividad, informalidad y bosques}

El nuevo Marco Macroeconómico Multianual ${ }^{14}$ 2021-2024 enfatiza la recuperación económica de los impactos del COVID-19, pero también se continuará impulsando el desarrollo de nuevos motores de crecimiento. ¿Podría ser el sector forestal uno de estos?

El MEF refiere que la presión tributaria del Perú (16.9\% del PBI en 2019) se encuentra rezagada respecto de Colombia (19.4\% del PBI), Ecuador (20.6\% del PBI) y Chile (21.1\% del PBI), así como del promedio de América Latina (23.2\% del PBI).

Asimismo, el MEF explícitamente menciona que se evaluará la creación de impuestos ambientales, citando la reciente publicación de la OCDE para política tributaria en tiempos de coronavirus, donde se indica que la crisis implica reflexionar en nuevas medidas tributarias y reconsiderar las tradicionales para brindar mayor progresividad al sistema tributario como un todo. Los países de la OCDE están dispuestos a considerar los impuestos al carbono, los impuestos de solidaridad, entre otros (OCDE 2020). No hay que olvidar que todos los países de la Alianza del Pacífico (Colombia, Chile y México) tienen impuestos a las emisiones de gases de efecto invernadero. En tiempos de pandemia parece más saludable gravar la emisión de estos gases que están muy relacionados a la contaminación del aire. 
Un impuesto a las emisiones de carbono, por más diminuto que fuera, sería suficiente para solventar una seria política a la inversión privada en el sector forestal como nuevo motor de crecimiento.

La Estrategia Nacional de Bosques y Cambio Climático (ENBCC) de 2016 menciona que cerca de 8 millones de hectáreas deforestadas pueden generar empleo mediante la producción agraria, agroforestal y las plantaciones forestales, mejorando la productividad de servicios ecosistémicos y conservar los bosques naturales. Esto generaría bionegocios, turismo, entre otros. Si se utilizara la mitad, esto es 4 millones de ha, se crearía 1 millón de empleos directos, sin contar los indirectos.

Para mejorar el desempeño ambiental del Perú, la OCDE realizó cinco recomendaciones para el sector agropecuario y gestión de los bosques ${ }^{15}$. Los más relevantes para bosques son: mejorar la gobernabilidad forestal y coordinación entre MINAM y MINAGRI, así como fortalecer la institucionalidad para acelerar la titulación de tierras. Los avances son la continuación de la implementación de la ENBCC, así como las acciones de la Declaración Conjunta de Intención con Noruega y Alemania para cumplir con el compromiso climático del Perú ${ }^{16}$ y la propia ENBCC.

La ENBCC enfatiza aumentar el valor de los bosques, vía manejo forestal sostenible, haciéndolo más competitivo frente a actividades que generan deforestación y degradación. Sin embargo, no tiene un texto específico para la inversión privada.

La Política Nacional Agraria aprobada en $2016^{17}$ propugna la conservación e incremento de los recursos forestales vía agroforestería, plantaciones forestales y otros, pero aún está pendiente la aprobación de una Política Nacional Forestal y de Fauna Silvestre. En tanto esta no se apruebe, siguen pendientes el Plan Nacional Forestal y de Fauna Silvestre, así como el Programa Nacional de Promoción de Plantaciones Forestales Comerciales, que eran metas de corto plazo (para 2018) establecidas para cumplir con las referidas recomendaciones de la OCDE ${ }^{18}$.

\footnotetext{
15 Se trata de las recomendaciones 51 a 55 que se resumen en: 51. robustecer la gobernabilidad forestal y mejorar las capacidades para el manejo sostenible de los recursos naturales. 52. Fortalecer la investigación agrícola considerando los retos del cambio climático. 53. Evaluar los efectos ambientales perjudiciales de los incentivos productivos. 54. Fortalecer el monitoreo de la calidad ambiental en el sector agropecuario (plaguicidas, educación) y 55. Fortalecer la institucionalidad responsable de la titulación de tierras. Ver todos los detalles y metas oficiales en el anexo del decreto supremo 005-2017-MINAM/Plan de Acción para implementar las recomendaciones de la evaluación de desempeño ambiental del Perú.

16 Contribuciones nacionalmente determinadas, NDC por sus siglas en inglés.

17 Decreto supremo 002-2016-MINAGRI.

18 Ver anexo del decreto supremo 005-2017-MINAM, Plan de acción para cumplir con las recomendaciones de la OCDE en lo referente a desempeño ambiental.
} 


\section{Situación actual de los bosques y otros compromisos del Perú}

En la siguiente tabla se puede ver que el $27 \%$ de los bosques en la Amazonia están en áreas naturales protegidas y $22 \%$ en comunidades nativas, campesinas y reservas indígenas. Los bosques de producción permanente para concesiones son el $25 \%$, pero de este porcentaje, más de 8 millones de ha son reservas para futuras concesiones. De los 10 millones de ha concesionadas, más de 4 millones han revertido al Estado por no estar operativas. De otro lado, es excesivo que 13 millones de ha de bosques no están caracterizados.

Tabla 3

Áreas de bosque por categorías territoriales

\begin{tabular}{|c|c|c|c|c|c|}
\hline \multicolumn{2}{|c|}{ CATEGORÍAS TERRITORIALES } & \multicolumn{2}{|c|}{ BOSQUE 2011} & \multicolumn{2}{|c|}{ BOSQUE 2018} \\
\hline & & ha & $\%$ & ha & $\%$ \\
\hline \multicolumn{2}{|l|}{ Áreas protegidas } & $19,177,606$ & 24.49 & $18,544,880$ & 27.10 \\
\hline \multicolumn{2}{|c|}{$\begin{array}{l}\text { Comunidades nativas y campesinas, } \\
\text { y reservas territoriales }\end{array}$} & $16,726,361$ & 21.36 & $15,081,636$ & 22.04 \\
\hline \multicolumn{2}{|c|}{$\begin{array}{l}\text { Bosques de producción } \\
\text { permanente en concesión }\end{array}$} & $10,553,552$ & 13.48 & $10,106,559$ & 14.77 \\
\hline \multicolumn{2}{|c|}{$\begin{array}{l}\text { BOSQUES DE PRODUCCIÓN } \\
\text { PERMANENTE EN RESERVA (futuras } \\
\text { concesiones o bosques locales) }\end{array}$} & $8,274,764$ & 10.57 & $7,633,570$ & 11.16 \\
\hline \multicolumn{2}{|l|}{ Predios rurales } & $1,962,931$ & 2.51 & 807,078 & 1.18 \\
\hline ZONA ESPECIAL & $\begin{array}{l}\text { Humedales en } \\
\text { la Amazonia }\end{array}$ & $3,237,617$ & 4.13 & $3,037,357$ & 4.44 \\
\hline \multicolumn{2}{|l|}{ No categorizado } & $18,372,710$ & 23.46 & $13,211,505$ & 19.31 \\
\hline \multicolumn{2}{|l|}{ TOTAL } & $78,305,541$ & 100.00 & $68,422,585$ & 100.00 \\
\hline
\end{tabular}

Cuadro resumido por la autora en base MINAM (2020b: 9).

El Perú ha comprometido, en el marco del Acuerdo de París sobre cambio climático, evitar y/o reducir el 35\% de sus emisiones de gases de efecto invernadero estimadas para 2030. El inventario más reciente indica que la deforestación es causante del 44.9\% de las emisiones nacionales de 2014. Por lo que el compromiso no se cumplirá si no se controla la deforestación. El promedio anual de deforestación es 150 mil ha anuales y la razón principal reside en la pequeña agricultura migratoria operada por colonos migrantes de los andes (SERFOR 2015: 15-ss).

Dado que un 10\% del compromiso depende de la cooperación internacional, 
con el objetivo de que estos países contribuyan a la reducción de las emisiones de GEI por deforestación y degradación forestal en el Perú.

De los 6 resultados comprometidos del DCI, se ha cumplido con el pago de transferencias directas condicionadas a comunidades nativas en más de 2 millones de hectáreas por la conservación de bosques. Los otros cinco resultados están en proceso, siendo el de menor avance el caracterizar el 50\% de más de 13 millones de ha de bosques. El avance es de apenas 4\%, y estas zonas presentan importantes niveles de deforestación. En tiempos de pandemia por el coronavirus, parece diminuta la cifra de 10 soles/ha/año que se viene abonando a las comunidades nativas por conservación de bosques desde 2013.

Otro resultado importante a considerar es el proceso de establecer una coalición público-privada con compañías multinacionales comprometidas con ambiciosas políticas de deforestación cero. Al respecto se tiene una hoja de ruta elaborada dirigida a la agricultura libre de deforestación. Asimismo, el Perú se ha unido a la Alianza de Bosques Tropicales (TFA por sus siglas en inglés) que promueve la asociación con el sector privado y la sociedad civil hacia una producción sostenible y cadenas de suministro de productos básicos libres de deforestación.

\section{Detener la deforestación y crear empleo}

Como afirma Marc Dourojeanni (2020), el sector agrario hace todo lo posible para aumentar la deforestación, y lo mismo sucede con el sector minero y transporte. Considerando que la Amazonia concentra los niveles de pobreza más altos del Perú, hace falta una política unitaria para su desarrollo sostenible.

El bosque bien puede ser uno de los nuevos motores de desarrollo económico. Dourojeanni sostiene que, si las plantaciones operan en tierras deforestadas, su producción reducirá la presión por explotar los bosques naturales, que solo producirían madera fina y de alto valor. Asimismo, propone que la reforestación dependa del sector agrario porque una plantación no se diferencia de una industrial o de fruta.

El impulso del desarrollo sostenible de la Amazonia requeriría el funcionamiento armónico de tres actores principales: las comunidades, los gestores de las áreas naturales protegidas y el sector privado en los bosques de producción. A través de las entrevistas realizadas para este artículo ${ }^{19}$ se puede percibir que la ausencia de inversión privada forestal de gran escala se debería a lo siguiente:

19 Para la redacción de este artículo, en la primera semana de setiembre se entrevistó a cuatro ex ministros del Ambiente y a un asesor financiero de inversiones extranjeras forestales. 
- Multiplicidad de instituciones sin una política forestal

En el caso del sector minero, una sola autoridad es competente desde la exploración hasta el procesamiento minero, sin embargo, en el sector forestal está MINAGRI/SERFOR, OSINFOR, Ministerio de la Producción, MINCETUR, MINAM y los Gobiernos Regionales.

- Legislación ambigua respecto de la deforestación y ausencia de titulación

La inseguridad jurídica se debe a que la mayor parte del territorio nacional no cuenta con títulos, ya sea tierra pública o privada. Esto es un obstáculo para cualquier tipo de inversión. Asimismo, existe la interpretación de «deforestación legal» en tierras agrícolas que solicitan autorización para desbosque en la Amazonia ${ }^{20}$.

- Sector de riesgo y rentabilidad a largo plazo Incendios forestales, invasiones, entre otros, pueden suceder en un negocio que no verá ganancias hasta después de ocho a diez años, dependiendo del producto.

- Financiamiento con altas tasas de interés para una inversión de largo plazo hacen el negocio inviable

Desde el punto de vista de la inversión privada para plantaciones forestales se estaría demandando dos temas: 1) poner en valor las tierras deforestadas de los bosques de producción permanente, ofreciendo a inversionistas de plantaciones forestales lotes de un mínimo de 8 mil ha, y 2) un fondo forestal a gran escala con otros inversionistas institucionales privados para otorgar financiamiento o realizar inversiones en el sector ${ }^{21}$. Lograr altos niveles de inversión privada resultaría en un control casi automático de la deforestación en zonas extensas, a la vez que se crearía una cantidad considerable de empleo formal.

20 La SPDA, según el Dr. José Luis Capella (comunicación personal, setiembre de 2020), viene litigando en torno a esta interpretación en algunos casos judiciales.

21 En entrevista con un asesor financiero experto en el tema (comunicación personal, setiembre de 2020), este sostuvo que «Al funcionario público y a la banca privada, por tema de riesgos, les cuesta entender la magnitud de las inversiones en el sector forestal. Por ejemplo, en la especia que pensamos plantar, el monto mínimo de inversión es de 50 millones de dólares, lo cual se traduce en plantar entre 8 mil a10 mil hectáreas». Ver mas información en diario Gestión (2018). 


\section{6. ¿Deforestación cero en la cadena de suministro?}

Por los preparativos a la COP26, Conferencia de la ONU sobre el Cambio Climático que se realizará en Glasgow/Reino Unido el próximo noviembre de 2021, se viene consultando un proyecto de ley británica cuyo objetivo es proteger los bosques tropicales y limpiar las cadenas de valor (DEFRA 2020). Toda gran empresa que opera en el Reino Unido estaría prohibida de usar productos cultivados en tierras deforestadas ilegalmente, para lo que deberían publicar información de dónde provienen sus materias primas, como en el caso del cacao y el caucho, por ejemplo. Además, deberán comprobar que se produjeron de acuerdo con las leyes locales que protegen los bosques.

Por su parte, la Unión Europea tiene bajo consulta una directiva similar con el objetivo de impedir que en la UE se puedan comercializar productos que contribuyan a la deforestación y a la destrucción de la biodiversidad

Queda claro entonces que el Perú tendrá que limar las ambigüedades legales y políticas respecto de las normas de titulación y deforestación en la Amazonia.

\section{Reflexiones finales y sugerencias para la investigación académica}

A lo largo de este artículo se puede concluir el rol que ha tenido el Ministerio de Economía y Finanzas en la decisión de políticas ambientales y el cumplimiento de compromisos internacionales con la OCDE y el Acuerdo de París. Es ostensible que el MEF ha carecido de visión de largo plazo, más aún si miramos el tema forestal. La crisis abre una posibilidad importante para que esto cambie pronto, ojalá con el impulso de los líderes empresariales que buscan una transformación basada en la naturaleza.

Mientras se procesa la gran decisión política de un plan de desarrollo sostenible de la Amazonia peruana en el marco del Bicentenario, es recomendable elaborar una agenda de investigación académica sobre los bosques en el Perú que tenga como pregunta básica si es posible controlar la deforestación mientras se crea empleo, se mitiga el cambio climático y se previene una próxima pandemia.

Algunas ideas para esta agenda de investigación académica, en parte considerando las medidas prioritarias aconsejadas por ex ministra(o)s de ambiente, serían las que a continuación formulamos como preguntas:

1. ¿Cuál es la hoja de ruta para concluir la titulación de comunidades nativas y otorgamiento de derechos a gran escala para el aprovechamiento sostenible de los bosques? 
Para esto, Dourojeanni (2020) aconseja dejar de lado el «reglamento de clasificación de tierras», dados los avances de la ciencia agropecuaria ${ }^{22}$. Hay opiniones contrapuestas de si es posible o no aglutinar tierras suficientes para plantaciones de reforestación ${ }^{23}$.

2. ¿Cómo y cuánto puede contribuir la inversión privada a detener la deforestación? ¿Cuáles serían las condiciones ideales para las plantaciones forestales, bionegocios y productos libres de deforestación en su cadena de suministro?

3. ¿Qué resulta necesario para concretar un ordenamiento participativo del territorio amazónico por cuencas construyendo confianza y diálogo entre las comunidades, el sector privado y el Estado? ¿Qué rol podrían tener las iniciativas interreligiosas en el proceso de crear confianza?

4. ¿Cómo lograr el financiamiento forestal a mínimas tasas de interés para hacer viable el negocio forestal? ¿Cuáles serían los vehículos financieros más adecuados para promover inversión privada forestal?

5. ¿Cómo debe concretarse la prohibición inmediata de derechos agrícolas sobre el bosque? Esto implicaría que ningún beneficiario de crédito del sector privado debería recibir financiamiento para deforestar.

6. Para el Perú, ¿cuál es la estrategia más adecuada para la certificación de productos básicos como el café, el cacao o la palma aceitera, considerando los esquemas voluntarios como obligatorios?

7. ¿Cómo ampliar los bionegocios con asociaciones entre comunidades y empresa privada que generen mayor valor al bosque en pie?

Importante notar otras prioridades señaladas por Dourojeanni (2020), como la moratoria de construcción de carreteras atravesando bosques naturales. Esta medida sería bien recibida si se da la regularización de la tenencia de tierra con una titulación eficiente. Esto tendría que darse en conjunción con medidas para mejorar la vida de los pueblos alejados

Ad portas del Bicentenario de la Independencia del Perú, corresponde pensar en positivo el futuro de la Amazonia peruana, reconociendo no solo el impacto positivo de la inversión privada forestal y la agroforestería, sino también el reconocimiento de los valores culturales de las comunidades y sus necesidades básicas. El consumo responsable y compromiso de las empresas para influenciar en la cadena de suministro de los productos libres de deforestación y pérdida de biodiversidad tienen un papel fundamental.

22 Dourojeanni (2020) agrega que con ello quedaría fuera el criterio legal de «suelos de aptitud forestal» y permitiría pasar a titular propiedades sobre esos suelos, incluyendo — si aún subsisten en ellas- bosquetes que deberán ser cuidados por los propios dueños. 


\section{Referencias}

Andreoni, V y Miola, A. (2016). Competitiviness and Sustainable Development Goals Publications Office of the European Union. https://op.europa. eu/en/publication-detail/-/publication/9ff7f616-c680-11e6-a6db01aa75ed71a1/language-en

Bar-On, Y, Philips, R. y Milo, R. (2018). The biomass distribution on Earth. Proceedings of the National Academy of Sciences, 115(25): 6506-6511. https://doi.org/10.1073/pnas.1711842115

Cann, 0. (2017). What exactly is economic competitiveness?. En World Economic Forum. https://www.weforum.org/agenda/2017/09/what-is-economiccompetitiveness/

ComunicacionesPerú 2021(23 deoctubre 2020).ValorCompartido:Avances en Perú (Michael Porter). [Video] https://www.youtube.com/watch?v=f_I15CP9GE\&list=PLmFYiE8u6u02-9-0eS90g5sLELCa1g2cE\&index=6\&t=758s

Conexión ESAN (15 de noviembre 2010). Lo que nos dejó la CADE 2010. https:// www.esan.edu.pe/conexion/actualidad/2010/11/15/lo-que-nos-dejo-lacade-2010/

DEFRA (2020). Due diligence on forest risk commodities Consultation document. https://consult.defra.gov.uk/eu/due-diligence-on-forest-riskcommodities/supporting_documents/duediligenceconsultationdocument. pdf

Dourojeanni, M. (2 de setiembre 2020). [Opinión] ¿Cómo combatir la deforestación? SPDA. Actualidad Ambiental. https://www.actualidadambiental.pe/ opinion-como-combatir-la-deforestacion/?utm_source=facebook\&utm medium=social\&utm_campaign=SocialWarfare

Dresch, A., Collatto, D. C. \& Lacerda, D. P. (2018). Theoretical understanding between competitiveness and productivity: firm level. Ingeniería y competitividad, 20(2): 69-86. https://dx.doi.org/10.25100/iyc.v20i1.5897

FAO (2019). Propuesta de incentivos para la inversión en recuperación de áreas degradadas. Sociedad Peruana de Derecho Ambiental Lima.

Guariguata, MR; Arce, J; Ammour, T y Capella, JL. (2017). Las plantaciones forestales en Perú: Reflexiones, estatus actual y perspectivas a futuro. Bogor, Indonesia: CIFOR.

Gestión (5 de marzo, 2018). Fondo Campbell afina detalles para plantación forestal de 8,000 hectáreas en Perú. Diario Gestión, sección Economía. https:// gestion.pe/economia/fondo-campbell-afina-detalles-plantacion-forestal8-000-hectareas-peru-228586-noticia/

Timber Committee (2018). Statement Regarding Implementation of the PTPA Forest Annex and Peru's July 2018 Verification Report. https://ustr.gov/ sites/default/files/Timber\%20Committee\%20Report\%2009.17.18.pdf

Iturregui, P. y Dutschke, M. (2005). Liberalisation of environmental goods \& services and climate change. HWWA DISCUSSION PAPER 335. Hamburgisches WeltWirtschafts-Archiv (HWWA) y Hamburg Institute of International Economics. Hamburg Institute of International Economics 2005 ISSN 1616-4814. 
OCDE (25 de junio 2009) Declaration on Green Growth. https://www.oecd.org/ env/44077822.pdf

OCDE (mayo 2020). Tax and Fiscal Policy in Response to the Coronavirus Crisis: Strengthening Confidence and Resilience. OCDE. http://www.oecd.org/ctp/ tax-policy/tax-and-fiscal-policy-in-response-to-the-coronavirus-crisisstrengthening-confidence-and-resilience.htm

Organización para la Cooperación Económica y Desarrollo (2020). Estudios de la OCDE sobre políticas públicas de conducta empresarial responsable: Perú. OCDE París.

PAGE (2017). Documento resumen. Perú: Crecimiento Verde. Análisis cuantitativo de políticas verdes en sectores seleccionados de la economía. https://www. un-page.org/files/public/analisis-cuantitativo-modelo-t21-y-politicasverdes-sectoriales.pdf

MINAM (2020b). Reporte de Cumplimiento de la Declaración Conjunta de Intención sobre REDD+ suscrita entre los Gobiernos de Perú, Noruega y Alemania al 2019 (borrador).

MINAM (abril 2020a). Segundo reporte de seguimiento y monitoreo del Plan de Acción para implementar las recomendaciones de la Evaluación de Desempeño Ambiental del Perú (EDA). https://sinia.minam.gob.pe/documentos/ segundo-reporte-seguimiento-monitoreo-plan-accion-implementar-las

MEF (2019). Plan Nacional de COMPETITIVIDAD Y PRODUCTIVIDAD 2019-2030 Aprobado por Decreto Supremo $N^{\circ}$ 237-2019-EF. DOCUMENTO RESUMEN https://www.mef.gob.pe/concdecompetitividad/Plan_Nacional_de_ Competitividad_y_Productividad_PNCP.pdf

MEF (26 agosto 2020) Marco Macroeconómico Multianual 2021-2024. En diario oficial El Peruano 28 de agosto.

Porter, M. E. y van der Linde, C. (1995). Toward a New Conception of the Environment-Competitiveness Relationship. Journal of Economic Perspectives, 9 (4): 97-118.

SERFOR (2015). Interpretación de la dinámica de la deforestación en el Perú y lecciones aprendidas para reducirla. Documento de trabajo. http:// siar.minam.gob.pe/puno/sites/default/files/archivos/public/docs/ interpretacion-de-la-dinamica-de-la-deforestacion.pdf

Simpleshow Foundation (2017). What is One Health? [Video]. https://www. youtube.com/watch?v=kfluP-tFC2k

Smil, V. (2016). Harvesting the biosphere. The World Financial Review.

United Nations Environment Programme and International Livestock Research Institute (2020). Preventing the next pandemic: Zoonotic diseases and how to break the chain of transmission. Nairobi. Kenya: UNEP. https:// www.unenvironment.org/resources/report/preventing-future-zoonoticdisease-outbreaks-protecting-environment-animals-and 